\title{
BMJ Open Digital auscultation as a novel childhood pneumonia diagnostic tool for community clinics in Sylhet, Bangladesh: protocol for a cross- sectional study
}

\author{
Salahuddin Ahmed (D) , ${ }^{1,2}$ Dipak Kumar Mitra, ${ }^{1,3}$ Harish Nair (1) ,2 \\ Steven Cunningham (1) , ${ }^{4}$ Ahad Mahmud Khan (D) ,, ${ }^{1,2}$ ASMD Ashraful Islam (1) , \\ Ian Mitra McLane, ${ }^{5}$ Nabidul Haque Chowdhury, ${ }^{1}$ Nazma Begum, ${ }^{1}$ \\ Mohammod Shahidullah, ${ }^{6}$ Muhammad Shariful Islam, ${ }^{7}$ John Norrie (D) , ${ }^{8}$ \\ Harry Campbell, ${ }^{2}$ Aziz Sheikh, ${ }^{2}$ Abdullah H Baqui, ${ }^{9}$ Eric D McCollum (1) 9,10
}

To cite: Ahmed S, Mitra DK, Nair $\mathrm{H}$, et al. Digital auscultation as a novel childhood pneumonia diagnostic tool for community clinics in Sylhet, Bangladesh: protocol for a crosssectional study. BMJ Open 2022;12:e059630. doi:10.1136/ bmjopen-2021-059630

- Prepublication history for this paper is available online. To view these files, please visit the journal online (http://dx.doi. org/10.1136/bmjopen-2021059630).

Received 06 December 2021 Accepted 18 January 2022

Check for updates

(C) Author(s) (or their employer(s)) 2022. Re-use permitted under CC BY. Published by BMJ.

For numbered affiliations see end of article.

Correspondence to Dr Salahuddin Ahmed; sahmed773@yahoo.com

\section{ABSTRACT}

Introduction The WHO's Integrated Management of Childhood IIInesses (IMCl) algorithm for diagnosis of child pneumonia relies on counting respiratory rate and observing respiratory distress to diagnose childhood pneumonia. IMCl case defination for pneumonia performs with high sensitivity but low specificity, leading to overdiagnosis of child pneumonia and unnecessary antibiotic use. Including lung auscultation in IMCI could improve specificity of pneumonia diagnosis. Our objectives are: (1) assess lung sound recording quality by primary healthcare workers ( $\mathrm{HCWs)} \mathrm{from} \mathrm{under-5} \mathrm{children} \mathrm{with} \mathrm{the}$ Feelix Smart Stethoscope and (2) determine the reliability and performance of recorded lung sound interpretations by an automated algorithm compared with reference paediatrician interpretations.

Methods and analysis In a cross-sectional design, community HCWs will record lung sounds of $\sim 1000$ under-5-year-old children with suspected pneumonia at first-level facilities in Zakiganj subdistrict, Sylhet, Bangladesh. Enrolled children will be evaluated for pneumonia, including oxygen saturation, and have their lung sounds recorded by the Feelix Smart stethoscope at four sequential chest locations: two back and two front positions. A novel sound-filtering algorithm will be applied to recordings to address ambient noise and optimise recording quality. Recorded sounds will be assessed against a predefined quality threshold. A trained paediatric listening panel will classify recordings into one of the following categories: normal, crackles, wheeze, crackles and wheeze or uninterpretable. All sound files will be classified into the same categories by the automated algorithm and compared with panel classifications. Sensitivity, specificity and predictive values, of the automated algorithm will be assessed considering the panel's final interpretation as gold standard.

Ethics and dissemination The study protocol was approved by the National Research Ethics Committee of Bangladesh Medical Research Council, Bangladesh (registration number: 09630012018) and Academic and
Strengths and limitations of this study

- Evaluating the quality of lung sound recordings in a first-level facility where auscultation is usually unavailable and challenging to obtain due to a typically crowded and noisy environment and providers may not get enough time to calm the child due to time pressure from a high-volume patient.

- This study will assess the feasibility of recording lung sounds by frontline community health workers who do not usually use conventional stethoscopes during clinical care.

- Two standardised paediatricians masked to the child's clinical status will independently classify the recorded lung sounds, and a third masked and independent paediatrician will arbitrate any discrepancies.

- A machine learning algorithm developed by Johns Hopkins and Sonavi Labs will detect abnormal lung sounds and be compared with classifications by human listeners/paediatricians.

- The study will not have chest radiography findings of enrolled children, which is considered by many a gold standard for pneumonia diagnosis, as chest radiography is not available at this level of the health system in Bangladesh.

Clinical Central Office for Research and Development Medical Research Ethics Committee, Edinburgh, UK (REC Reference: 18-HV-051). Dissemination will be through conference presentations, peer-reviewed journals and stakeholder engagement meetings in Bangladesh. Trial registration number NCT03959956.

\section{INTRODUCTION}

Childhood pneumonia is one of the leading causes of death in children younger than 5 years globally ${ }^{1}$ and accounts for an 
estimated 0.8 million deaths in children annually. ${ }^{2}$ The WHO estimates that the African and the South-East Asian Regions contribute to more than $75 \%$ of total paediatric deaths from pneumonia. ${ }^{3}$ It is estimated that the annual incidence of childhood pneumonia in low- and middleincome countries (LMICs) is 231 episodes per 1000 children. ${ }^{3}$ Pneumonia is also a significant cause of hospitalisation, ${ }^{4}$ and about 16.4 million children in LMICs were hospitalised due to pneumonia in $2015 .^{3}$ A populationbased study in Bangladesh estimated that the annual incidence of pneumonia was 360 episodes per 1000 childyears, of which $7.3 \%$ were hospitalised. ${ }^{5}$ The case-fatality rate of child pneumonia in Bangladesh is estimated to be $2 \%-4 \%{ }^{67}$

The WHO and UNICEF Integrated Management of Childhood Illness (IMCI) guidelines have been the foundation of pneumonia management in LMICs since mid-90s. ${ }^{8}$ Per current IMCI guidelines, a child with fast breathing and/or chest indrawing without any danger sign is classified as non-severe pneumonia and treated at home with oral antibiotics. ${ }^{10}$ These guidelines have proven to be one of the most important childhood pneumonia interventions for LMICs to date, and up to $36 \%$ of the under-5-year-old mortality reductions in LMICs have been attributed to guideline implementation. ${ }^{11-15}$

Despite its overall success, the IMCI algorithm for diagnosis of child pneumonia can still be improved. First, the guidelines were developed when access to vaccines was limited and child pneumonia mortality was high, so the guidelines intentionally prioritised sensitivity over specificity to ensure children with possible bacterial disease received antibiotics. ${ }^{810}$ Thus, the IMCI algorithm overdiagnoses many children with pneumonia who do not require antibiotics and they may have different treatable diseases. ${ }^{16}{ }^{17}$ Second, the WHO guidelines do not include lung auscultation in their pneumonia definition for frontline healthcare workers despite lung auscultation serving as the cornerstone for pneumonia diagnosis in most ambulatory, well-resourced settings staffed by clinicians trained to perform lung auscultation. ${ }^{10}$ The exclusion of auscultation findings in these guidelines likely stems from its high interobserver variability and subjectivity, regardless of the training level of healthcare providers, and the related difficulty in training healthcare workers to effectively use a conventional stethoscope. ${ }^{18-22}$ Traditional stethoscopes themselves are also limiting, attenuating higher frequency sounds, like wheezing and crackles, yet transmitting ambient noises and tubular resonance effects. ${ }^{202324}$ Automated real-time classification of lung sounds or digital auscultation may overcome these limitations. ${ }^{25}$ Digital auscultation augmented by artificial intelligence algorithms has the potential to be a highly specific respiratory diagnostic tool feasible for use by front line healthcare workers in LMICs. Operationally, the inclusion of adventitious sound classifications in current IMCI guidelines could help to reduce ${ }^{26}$ unnecessary use of antibiotics in LMICs.

A digital stethoscope can convert an acoustic sound to electronic signals, which can be further amplified for optimal listening. These electronic signals can then be processed and digitalised to transmit to a personal computer or a laptop. ${ }^{27}$ Automatic lung sound analysis, aiming to overcome the limitations of conventional auscultation, has been the recent focus of a significant amount of research, and some commercial systems are already available in the market. ${ }^{28} 29$

The digital stethoscope has advantages over the analogue stethoscope in different stages of auscultation. An analogue stethoscope requires the proper placing of the diaphragm or bell in the correct positions of the human body to listen to internal body sounds. More modern digital stethoscopes do not necessarily require exact placement for two reasons. First, they convert the acoustic wave into electric signals and replace the doublesided chest piece (diaphragm and bell) with transducers to convert acoustic signals to electric signals. Second, the chest piece is packed with transducer arrays to achieve a uniform sensitivity over the entire active area. Together, this design delivers a strong signal even when the chest piece is not placed in precisely the right position. ${ }^{3031}$ This advantage is essential for minimally trained healthcare providers.

Conventional auscultation using an analogue stethoscope also requires a quiet environment, and ideally with the patient in a quiet, cooperative state, which is difficult in hospitals/clinics and especially hospitals/clinics in LMICs where the number of patients are typically usually higher than capacity. This often results in patient examination rooms filled with chattering people, ringing phones and whirring fans; most importantly, the child being examined may then be agitated, uncooperative and/ or crying. Digital stethoscopes could improve listening capability through the use of noise cancelling technologies. ${ }^{32} 33$ Limitations of the human auditory system, which ranges from $20 \mathrm{~Hz}$ to $20 \mathrm{kHz}$ for young adults and the range shrinks after middle age,${ }^{34}$ are also a drawback in conventional auscultation using a conventional stethoscope. A digital stethoscope can amplify sounds up to 100 -fold. ${ }^{35}$ Much experience is required to interpret body sounds, and inter-rater variability is high regardless of healthcare providers' training level. ${ }^{21}$ Machine learning techniques can be used in digital auscultation to autoanalyse sounds ${ }^{28}$ and produce a diagnosis or treatment decision. $^{23}$

Johns Hopkins University and Sonavi Labs developed a novel digital stethoscope named Feelix Smart Stethoscope (figure 1), ${ }^{30} 31$ which improves lung signal strength by uniformly distributing highly sensitive microphones in an array pattern across the stethoscope diaphragm to increase the sensitivity and provide broader frequency response, a critical feature for identifying higher frequency pathologic lung sounds. Its $3.7 \mathrm{~V}$ and 250 amps/hour rechargeable battery can power $>20$ hours of use, important in rural communities with unreliable electricity. The device mitigates movement artefact and tubular resonance by using an ergonomic design to better secure the device on the child's chest. It also eliminates 


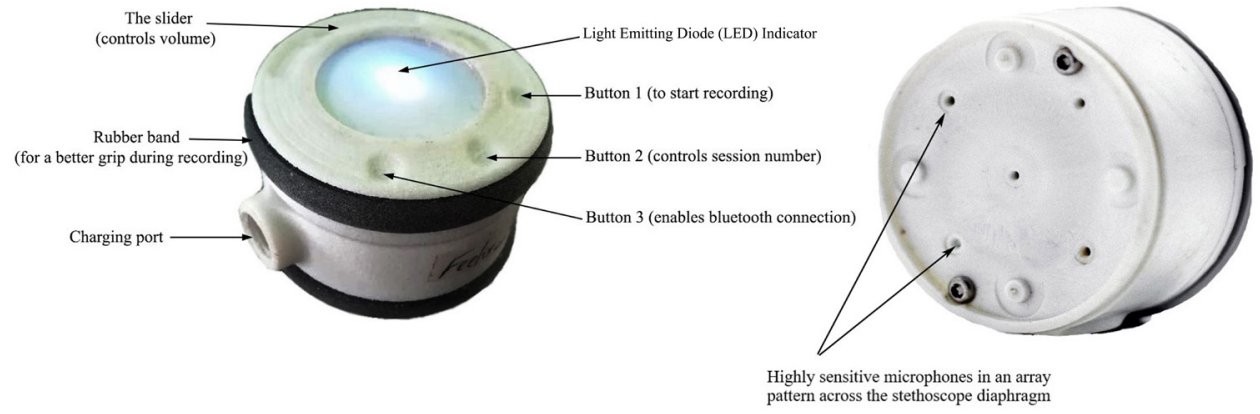

Figure 1 Feelix Smartscope.

the rubber stethoscope tubing, a source of ambient noise and friction contamination. Notably, the device includes an integrated external facing microphone that captures simultaneous environmental noise to the lung sound recording and removes the unwanted ambient noises through an adaptive spectral subtraction schema. ${ }^{24} 36$ The Feelix Smart Stethoscope also permits onboard data storage with a micro SD card. The device turns on when the user picks up the device or touches the top of the device. The device turns off automatically after $60 \mathrm{~s}$ when it does not sense any touch. The device has three touch buttons-button 1 to start recording, button 2 to start a new session to record a new child's four chest points recording, and button 3 to establish a Bluetooth connection with a mobile phone or tablet. It also has a slider to control sound volume level. In each session, the device automatically records $10 \mathrm{~s}$ for each four chest points of a child. The Feelix Smart Stethoscope has been successfully validated in the laboratory against six other commercially available electronic stethoscopes-including the Littmann 3200 electronic stethoscope and Thinklabs-and has demonstrated comparable results. ${ }^{37} 38$ Johns Hopkins and Sonavi Labs developed a machine learning algorithm that can provide an automated classification of adventitious lung sounds. This Feelix Smart Stethoscope and the machine learning algorithm will be used in this study.

\section{Objectives and hypotheses}

The primary objectives of the study are:

i. to assess whether lung sounds recorded using the Feelix Smart Stethoscope in children by health workers at community-level health facilities meet predefined quality thresholds established by experts; and

ii. to determine the reliability and performance of the Smartscope Respiratory Detector automated analysis algorithm on lung sounds recorded by community healthcare provider (CHCP) using the Smartscope, compared with reference interpretations by a paediatric listening panel.

The study hypotheses are:

i. More than $50 \%$ of patients will have 'quality' lung sound recordings (targeted goal), defined as at least $75 \%$ interpretable lung sound segments per patient (ie, 3 out of 4 chest positions). ii. The agreement between automated computerised analysis (respiratory detector) and paediatric listening panel will be high (kappa $>0.8$ ).

\section{METHODS AND ANALYSIS}

\section{Study setting}

This study will be implemented in the Projahnmo field site, a site for maternal, new-born and child health research, which was established in 2001 in Sylhet district of Bangladesh by a partnership of Johns Hopkins University, the Bangladesh Ministry of Health and Family Welfare (MOHFW) and several Bangladeshi institutions, including non-government organisations and academia. A well-established routine community-based pregnancy, birth and under-5 child surveillance system are being maintained by trained female community health workers (CHWs) in this site. The CHWs identify sick children during routine household visits and refer the children to the health facilities. They also educate carers of children about pneumonia signs and symptoms, so that the carers can visit nearby community clinic (CC) without delay.

Bangladesh has established about 13000 CCs, one each for $\sim 6000$ people. ${ }^{39}$ Each CC is staffed by a CHCP with at least 12th grade education and 3 months of preservice training, including IMCI guidelines. Each CHCP is responsible for providing primary healthcare for the population, including its catchment area's children. Nine CCs will be purposively selected from the Projahnmo surveillance area (Zakiganj sub-district of Sylhet district of Bangladesh) (figure 2). CHCP of respective CC will screen all under-5 children while providing primary healthcare.

All CHWs and CHCPs will be trained and standardised to identify signs and symptoms of pneumonia according to WHO IMCI guidelines. A study physician will be recruited for providing training and supervision of CHCPs and CHWs in clinical assessments, measurement of peripheral oxyhaemoglobin saturation $\left(\mathrm{SpO}_{2}\right)$ and recording of lung sounds using the Smartscope. However, the study physician will not be directly involved in recording lung sound from the study participants. 


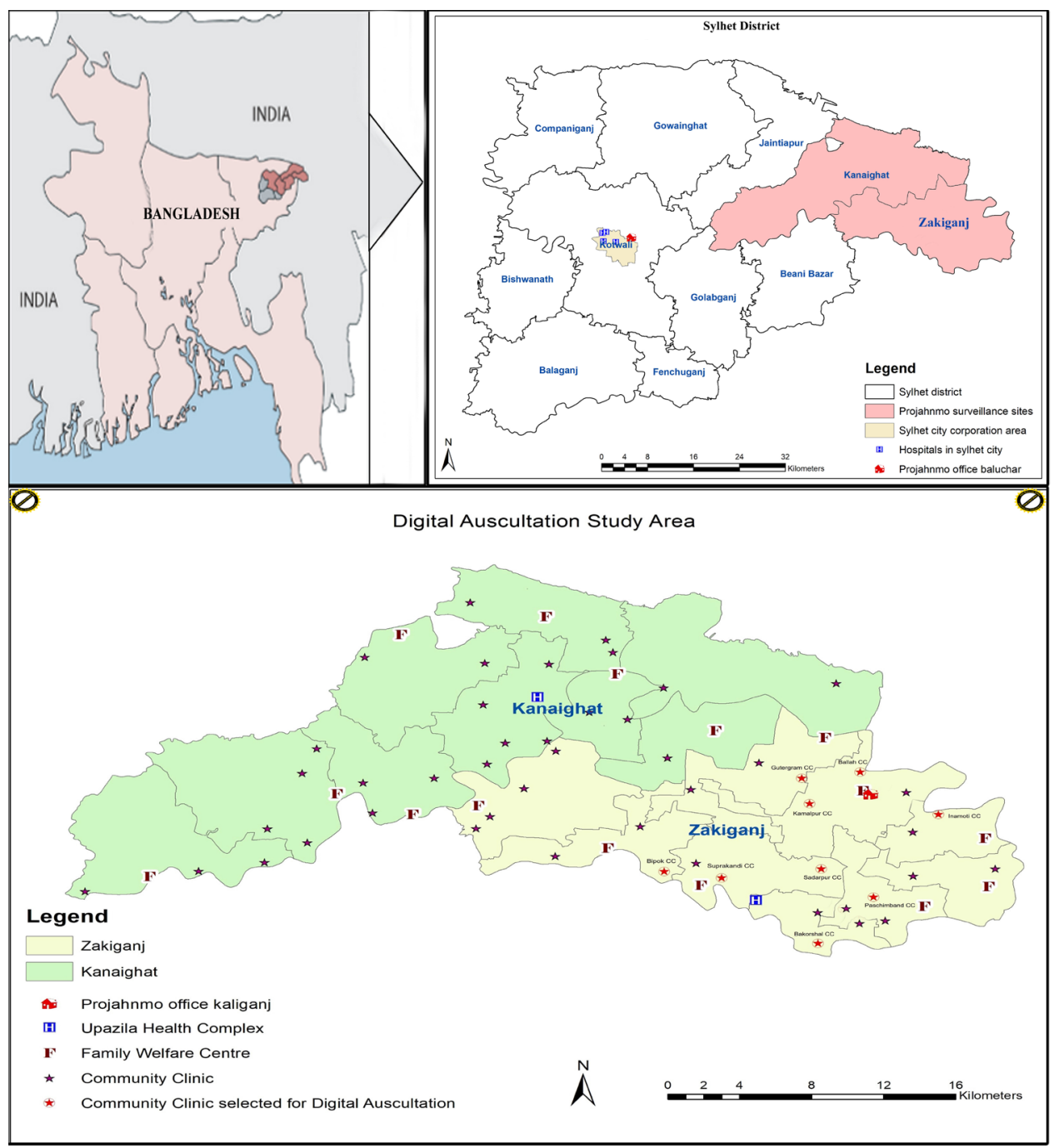

Figure 2 Study site.

\section{Study design and procedure}

Using a cross-sectional design, each CHCP will screen all children younger than 5 years consecutively visiting the CC. CHCP will obtain consent and record lung sounds of children who will fulfil the following criteria: history and/or observed cough and/or history and/or observed difficult breathing and a permanent resident of the Projahnmo site and not enrolled in the study within the past 30 days. CHCP will record sounds from four chest locations-two from the back and two from the front (figure 3). Each position will be recorded for approximately $10 \mathrm{~s}$ and the overall recording process will take about $1 \mathrm{~min}$. The recorded sound files will be then transferred to a password-protected server. CHCP will also examine the child for fast breathing by manually counting respirations and observe for abnormal breathing patterns such as lower chest wall indrawing, nasal flaring, head nodding, tracheal tugging, grunting, intercostal retractions and stridor when calm. CHCP will measure the $\mathrm{SpO}_{2}$ using a Masimo Rad5 pulse oximeter, temperature using a digital thermometer and anthropometry (weight, height and mid-arm circumference) using standard tools and techniques. The $\mathrm{SpO}_{2}$ data will be used to classify pneumonia according to IMCI guidelines. If any child has a $\mathrm{SpO}_{2}<90 \%$, then referral to the subdistrict health centre or Sylhet Osmani Medical College Hospital will be initiated. All enrolled children will be assessed after day 8 of enrolment by Projahnmo CHWs for treatment compliance and treatment outcome.

A total of 11 paediatricians will be trained to the methodology developed and validated during the Pneumonia Etiology Research for Child Health (PERCH) study. ${ }^{40}$ Only paediatricians successfully standardised to the methodology will serve as human listening panel members. Panellists will classify the recorded sound files of all four positions separately into five categories, for example (1) no wheeze and no crackles, (2) wheeze only (no crackles), (3) crackles only (no wheeze), (4) both wheeze and crackles or (5) uninterpretable. Two primary listeners will independently classify the recorded lung sounds, and any discrepancies will be arbitrated by the third listener (EDM). The panellists and EDM will be blinded to the clinical information of the children. Johns Hopkins and Sonavi Labs developed a machine learning algorithm; all 
Lung sound recording positions
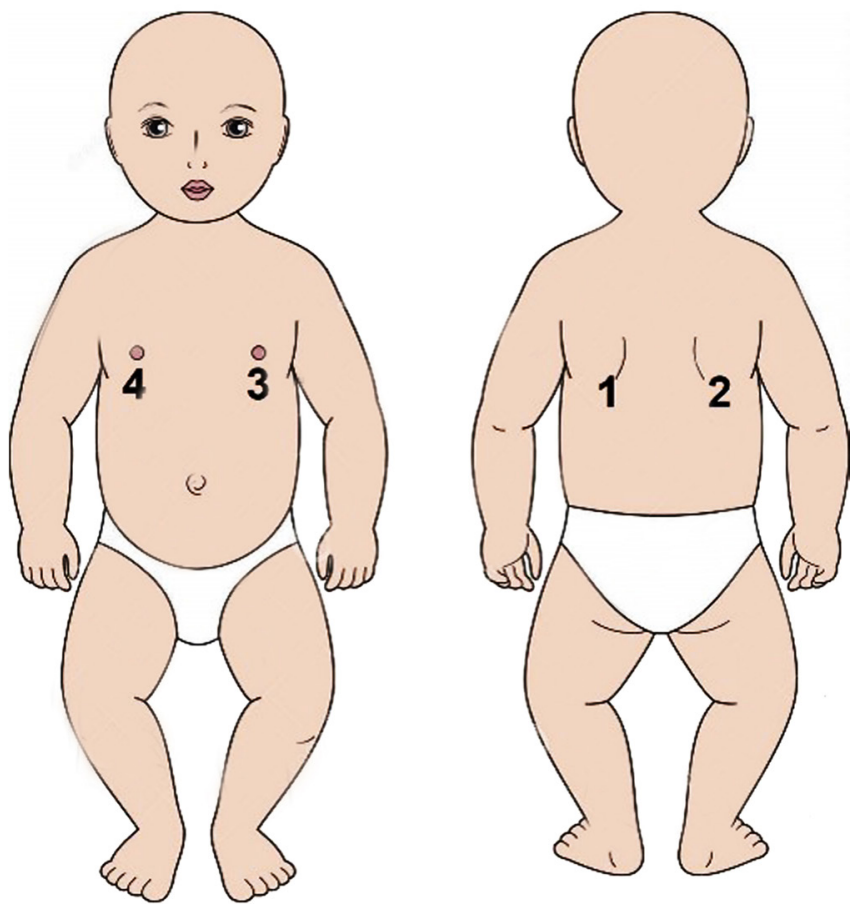

Figure 3 Lung sounds recording positions.

sound files will also be classified the same categories using this algorithm. This algorithm does not include the clinical information of the children and this will be blinded to the panel classification. Finally, both classification will be compared. Figure 4 depicts the study flow.

\section{Study flow}

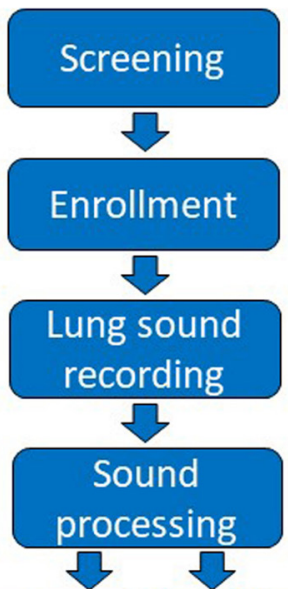

\section{Interpretation by} listening panel

\section{Comparison}

\section{Sample size calculation}

Cohen's kappa will be used to assess interlistener and computer-human agreement in this study. To detect a true kappa of 0.50 compared with a kappa of 0.40 under null hypothesis and assuming two-sided type I error of $5 \%$, power of $80 \%$ and two categories of frequencies equal to $30 \%$ and $70 \%$ normal and abnormal findings, respectively, a sample size of 752 will be required. ${ }^{41}$ This sample size has been calculated in Power Analysis and Sample Size software V.11.

We will include all quality recordings for this assessment. A quality recording is defined as a recording with $>75 \%$ interpretable chest positions (ie, three of the four chest positions recorded are interpretable). Assuming that $75 \%$ of all recordings will be of acceptable quality, we will need 1003 subjects to be enrolled in this study to obtain our required sample size of 752 .

\section{Statistical analysis}

A point estimate for the proportion of recordings along with a $95 \%$ CI that meets the definition of quality will be determined. The numerator will be the total number of recordings that were interpreted to meet quality criteria and the denominator will be the total number of recordings collected and interpreted. A raw agreement percentage will be determined between the interpretations of the two primary human listeners of the listening panel. The numerator will be the total number of recordings with an interpretation from the first and second human listener that agree, and the denominator will be the total number of recordings that have a final human interpretation from both primary listeners. Cohen's kappa, a metric that assesses the agreement beyond chance will be calculated, and a kappa adjusted for prevalence and bias will also be calculated. A kappa above 0.8 will be defined as perfect agreement, $0.61-0.8$ as high agreement, $0.41-0.6$ as moderate agreement, $0.21-0.4$ as fair agreement, $0.01-0.2$ as low agreement and 0 as no agreement.

A raw agreement percentage between the overall human listening panel's final interpretation result and the computerised analysis algorithm's final interpretation result will be determined. The numerator will be the total number of recordings with an interpretation from the human listening panel and the computerised algorithm that agree, and the denominator will be the total number of recordings that have both a human and computerised interpretation. Cohen's kappa, a metric that assesses the agreement beyond chance will be calculated, and a kappa adjusted for prevalence and bias will also be calculated. The same kappa scale detailed previously will be used for interpretation.

The performance of the computerised interpretation algorithm will be evaluated when assuming the human listening panel's final interpretation is the gold standard. Sensitivity, specificity, positive predictive value, negative predictive value and positive and negative likelihood ratios will be assessed. 


\section{Patient and public involvement}

We formed patient public involvement groups (PPIG) consisting of CHCPs, CHWs, Health Assistants, Physicians, community leaders, religious leaders, parents of under-5 children, teachers, local journalists and organised several meetings in the community for their insights, approval and support to implement the study. They believe its feasible and acceptable to implement the study. We also consulted and engaged several district and nationallevel stakeholders including public health programme managers, policymakers, paediatricians, physicians and civil society representatives during the development of the protocol and implementation strategy of the study. A technical review committee (TRC) consisting of policymakers and technical experts formed by the Bangladesh MOHFW reviewed and approved the protocol.

The study results will be reviewed by the PPIG and TRC before publishing in peer-reviewed journals, will be presented at international conferences and to health officials in Bangladesh. The findings also will be disseminated to stakeholders at Zakiganj subdistrict, Sylhet district and national level at Dhaka, Bangladesh.

\section{Data collection and storage}

Data will be collected using password-protected electronic devices (Samsung Galaxy Tab A V.7.0) in the Android platform. The data will be transferred to a server (SQL Server 2008 R2) located at Sylhet, Bangladesh in real-time using internet connectivity and will keep a backup copy daily in another server at the study Dhaka office. All the tablets and servers will be password protected. Recorded lung sounds will be transferred to the server. Paediatric listening panel members will fill up the lung sound interpretation on an online database, which will also be stored on the server in real time. Data collection has been started on 7 November 2019 and this is planned to be completed by March 2022.

\section{Ethics and dissemination}

Ethical approval was obtained from the National Research Ethics Committee of Bangladesh Medical Research Council, Bangladesh (Registration Number: 09630012018), and Academic and Clinical Central Office for Research and Development Medical Research Ethics Committee NHS, Lothian, Edinburgh, UK (REC Reference: 18-HV-051). This study was registered with ClinicalTrails.gov (NCT03959956). Informed written consent will be obtained from the parent or guardian of each child. Access to collected data will be restricted to individuals from the research team treating the participants, representatives of the sponsor(s) and representatives of regulatory authorities. Dissemination will be through conference presentations, peer-reviewed journals and stakeholder engagement meetings in Bangladesh. Anonymised data files will also be stored securely in the DataStore repository at the University of Edinburgh, UK and will be shared after publication of main paper.

\section{DISCUSSION}

This study aims to demonstrate the feasibility of collecting quality lung sounds by frontline health workers and to examine the performance of the machine learning algorithm against a panel of human listeners for identifying adventitial lung sounds. To our knowledge, this is the first study that will assess the recording of lung sounds of children younger than 5 years in first-level facilities by frontline workers in LMICs, where the burden of pneumonia and antibiotic use is high and diagnostic capacity is limited. The PERCH study enrolled children at a hospital setting and digital auscultation was performed by physicians, formally trained clinical assistants, or nurses, ${ }^{40}$ another study enrolled children at a tertiary level centre in Lima, Peru, ${ }^{42}$ and paediatricians recorded lung sounds in two tertiary level teaching hospitals in a study in Nepal. ${ }^{43}$ Recording lung sounds in a first-level facility poses unique challenges in that clinics are typically crowded and the environment can be chaotic and noisy. Furthermore, ill children younger than 5 years of age can be especially uncooperative in uncomfortable ambulatory settings, leading to unique challenges like agitation, crying, vocalisations and the associated auscultation artefacts these create. Healthcare providers in ambulatory settings may lack the necessary time to calm the child and address these issues due to pressures from a high patient volume. If frontline health workers can effectively use digital auscultation in their typical clinical setting, then many false-positive pneumonia cases may be spared from treatment with antibiotics, which may reduce the cost of treatment as well as reduce the chance of developing antimicrobial resistance. A systematic analysis of 132 national surveys from 73 countries reported that on average, 4 of 10 ill children below age 5 years in LMICs are treated with antibiotics. ${ }^{44}$ Recently, United Nations General Assembly announced that antimicrobial resistance is the most important and urgent threat globally. ${ }^{45}$

One limitation of our study is that chest radiography will not be performed as it is not routinely available in first-level facilities in Bangladesh, and we will not compare radiographic imaging with lung sound classification. Instead, lung sound classifications will be compared with clinical findings among children meeting IMCI pneumonia to those who do not. Lack of a gold standard reference for pneumonia is well-understood, and it is widely accepted that chest radiography itself lacks diagnostic accuracy for pneumonia. ${ }^{46}$ For example, it was found that many children with IMCI defined clinical pneumonia (age-specific tachypnoea) had normal chest radiographs ${ }^{47}$ and may have normal lung sounds and may not require antibiotics. In this study, we will utilise the paediatrician listening panel as our gold standard reference as previously described. ${ }^{40}$ We have shown that lung sound classifications of digital auscultation recordings generated using a listening panel approach have strong associations with radiographic findings and also mortality outcomes. $^{48}$ 
Future work ranges from additionally refining this device, based on lessons learnt from the use of the device at the first-level facility during this study as well as phases 1 and 2 clinical trials that prospectively integrate the digital auscultation into the WHO pneumonia management pathway to evaluate patient outcomes after digital auscultation-based decision-making. Eventually, if these preliminary studies are successful, a large multicountry clinical trial could be designed to evaluate the safety and efficacy of digital auscultation to improve pneumonia management.

\section{Author affiliations}

${ }^{1}$ Projahnmo Research Foundation, Dhaka, Bangladesh

${ }^{2}$ Usher Institute, The University of Edinburgh, Edinburgh, UK

${ }^{3}$ Public Health, North South University, Dhaka, Bangladesh

${ }^{4}$ Department of Child Life and Health, Royal Hospital for Sick Children, Edinburgh, UK

${ }^{5}$ Sonavi Labs, Baltimore, Maryland, USA

${ }^{6}$ Department of Neonatology, Bangabandhu Sheikh Mujib Medical University, Dhaka, Bangladesh

${ }^{7}$ Directorate General of Health Services, Ministry of Health and Family Welfare, Government of Bangladesh, Dhaka, Bangladesh

${ }^{8}$ Usher Institute, Edinburgh Clinical Trials Unit, University of Edinburgh No. 9,

Bioquarter, Edinburgh, UK

${ }^{9}$ Department of International Health, Johns Hopkins University Bloomberg School of Public Health, Baltimore, Maryland, USA

${ }^{10} \mathrm{Global}$ Program in Pediatric Respiratory Sciences, Eudowood Division of Pediatric Respiratory Sciences, Department of Pediatrics, Johns Hopkins School of Medicine, Baltimore, Maryland, USA

\section{Twitter Eric D McCollum @tinylungsglobal}

Acknowledgements The authors are grateful for the support and contributions of Dr Arunangshu Dutta Roy, Dr Arifa Islam, Dr Iffat Ara Jaben, Md. Shafiqul Islam, Asim Nehal, Dr. Md. Shamsul Haque, Dr. Himangshu Lal Roy, Dr. Premananda Mondol, Dr. Md. Jahurul Islam, Dr. Sabina Ashrafee Lipi, Dr. Abdullah Al Mehedi, Dr. Husam Md. Shah Alam and the Ministry of Health and Family Welfare, Government of Bangladesh.

Contributors EDM, SA and AHB conceptualised and designed this study. AHB, EDM, HN, SC provided mentorship to SA. HC, AS and DKM critically review the study design. JN and DKM reviewed the sample size estimation and analysis plan. EDM will provide training to the human listening panel and IMM will analyse the recorded sound files using a machine learning algorithm. NHC and NB will be responsible for data management and AAI will manage the field implementation of this study. SA drafted the manuscripts and all authors critically reviewed and approved the final manuscript before submission.

Funding This research was funded by the UK National Institute for Health Research (NIHR) (Global Health Research Unit on Respiratory Health (RESPIRE); Grant number 16/136/109) using UK aid from the UK Government to support global health research. The views expressed in this publication are those of the author(s) and not necessarily those of the NIHR or the UK Government. The RESPIRE collaboration comprises the UK Grant holders, Partners and research teams as listed on the RESPIRE website (https://www.ed.ac.uk/usher/respire) including Sian Williams.

Map disclaimer The inclusion of any map (including the depiction of any boundaries therein), or of any geographic or locational reference, does not imply the expression of any opinion whatsoever on the part of BMJ concerning the legal status of any country, territory, jurisdiction or area or of its authorities. Any such expression remains solely that of the relevant source and is not endorsed by BMJ. Maps are provided without any warranty of any kind, either express or implied.

Competing interests IMM will be paid for developing Feelix Smart Stethoscope and a machine learning algorithm from Sonavi Labs.

Patient and public involvement Patients and/or the public were involved in the design, or conduct, or reporting, or dissemination plans of this research. Refer to the Methods section for further details.

Patient consent for publication Consent obtained from parent(s)/guardian(s)
Provenance and peer review Not commissioned; externally peer reviewed.

Open access This is an open access article distributed in accordance with the Creative Commons Attribution 4.0 Unported (CC BY 4.0) license, which permits others to copy, redistribute, remix, transform and build upon this work for any purpose, provided the original work is properly cited, a link to the licence is given, and indication of whether changes were made. See: https://creativecommons.org/ licenses/by/4.0/.

\section{ORCID iDs}

Salahuddin Ahmed http://orcid.org/0000-0001-6771-0638

Harish Nair http://orcid.org/0000-0002-9432-9100

Steven Cunningham http://orcid.org/0000-0001-7342-251X

Ahad Mahmud Khan http://orcid.org/0000-0002-4347-0825

ASMD Ashraful Islam http://orcid.org/0000-0003-1597-4847

John Norrie http://orcid.org/0000-0001-9823-9252

Eric D McCollum http://orcid.org/0000-0002-1872-5566

\section{REFERENCES}

1 Liu L, Oza S, Hogan D, et al. Global, regional, and national causes of under-5 mortality in 2000-15: an updated systematic analysis with implications for the sustainable development goals. Lancet 2016;388:3027-35.

2 UNICEF StC, Every Breath Counts. Every child's right to survive: a 2020 agenda to end pneumonia deaths (Online), 2020. Available: https://www.unicef.org/reports/every-childs-right-survivepneumonia-2020 [Accessed 23 Oct 2021].

3 McAllister DA, Liu L, Shi T, et al. Global, regional, and national estimates of pneumonia morbidity and mortality in children younger than 5 years between 2000 and 2015: a systematic analysis. Lancet Glob Health 2019;7:e47-57.

4 Cilla G, Oñate E, Perez-Yarza EG, et al. Viruses in communityacquired pneumonia in children aged less than 3 years old: high rate of viral coinfection. J Med Virol 2008;80:1843-9.

5 Havers FP, Fry AM, Goswami D. Population-Based incidence of childhood pneumonia associated with viral infections in Bangladesh. Pediatr Infect Dis J 2018.

6 Naheed A, Saha SK, Breiman RF, et al. Multihospital surveillance of pneumonia burden among children aged $<5$ years hospitalized for pneumonia in Bangladesh. Clin Infect Dis 2009;48 Suppl 2:S82-9.

7 Saha S, Hasan M, Kim L, et al. Epidemiology and risk factors for pneumonia severity and mortality in Bangladeshi children $<5$ years of age before 10-valent pneumococcal conjugate vaccine introduction. BMC Public Health 2016;16:1233.

8 World Health Organization. Technical bases for the who recommendations on the management of pneumonia in children at first-level health facilities 1991.

9 Tulloch J. Integrated approach to child health in developing countries. Lancet 1999;354 Suppl 2:SIl16-20.

10 World Health Organization. Who IMCI chart booklet 2014. Geneva: World Health Organisation, 2014.

11 Theodoratou E, Al-Jilaihawi S, Woodward F, et al. The effect of case management on childhood pneumonia mortality in developing countries. Int J Epidemiol 2010;39 Suppl 1:i155-71.

12 Arifeen SE, Hoque DME, Akter T, et al. Effect of the integrated management of childhood illness strategy on childhood mortality and nutrition in a rural area in Bangladesh: a cluster randomised trial. The Lancet 2009;374:393-403.

13 Chowdhury EK, El Arifeen S, Rahman M, et al. Care at first-level facilities for children with severe pneumonia in Bangladesh: a cohort study. Lancet 2008;372:822-30.

14 Armstrong Schellenberg JRM, Adam T, Mshinda $\mathrm{H}$, et al. Effectiveness and cost of facility-based integrated management of childhood illness (IMCI) in Tanzania. Lancet 2004;364:1583-94.

15 Sazawal S, Black RE, Pneumonia Case Management Trials Group. Effect of pneumonia case management on mortality in neonates, infants, and preschool children: a meta-analysis of community-based trials. Lancet Infect Dis 2003;3:547-56.

16 Nantanda R, Tumwine JK, Ndeezi G, et al. Asthma and pneumonia among children less than five years with acute respiratory symptoms in Mulago Hospital, Uganda: evidence of under-diagnosis of asthma. PLoS One 2013;8:e81562.

17 Ellington LE, Najjingo I, Rosenfeld M, et al. Health workers' perspectives of a mobile health tool to improve diagnosis and management of paediatric acute respiratory illnesses in Uganda: a qualitative study. BMJ Open 2021;11:e049708. 
18 Margolis PA, Ferkol TW, Marsocci S, et al. Accuracy of the clinical examination in detecting hypoxemia in infants with respiratory illness. $J$ Pediatr 1994;124:552-60.

19 Murphy RLH, Vyshedskiy A, Power-Charnitsky V-A, et al. Automated lung sound analysis in patients with pneumonia. Respir Care 2004;49:1490-7.

20 Grenier MC, Gagnon K, Genest J, et al. Clinical comparison of acoustic and electronic stethoscopes and design of a new electronic stethoscope. Am J Cardiol 1998;81:653-6.

21 Brooks D, Thomas J. Interrater reliability of auscultation of breath sounds among physical therapists. Phys Ther 1995;75:1082-8.

22 Giłrup T, Bugge PM, Jensen AM. Interobserver variation in assessment of respiratory signs. physicians' guesses as to interobserver variation. Acta Med Scand 1984;216:61-6.

23 Gurung A, Scrafford CG, Tielsch JM, et al. Computerized lung sound analysis as diagnostic aid for the detection of abnormal lung sounds: a systematic review and meta-analysis. Respir Med 2011;105:1396-403.

24 Rennoll V, McLane I, Emmanouilidou D. Electronic stethoscope filtering mimics the perceived sound characteristics of acoustic stethoscope. IEEE journal of biomedical and health informatics 2020;25:1542-9.

25 Emmanouilidou D, McCollum ED, Park DE, et al. Computerized lung sound screening for pediatric auscultation in noisy field environments. IEEE Trans Biomed Eng 2018;65:1564-74.

26 Palafox M, Guiscafré H, Reyes H, et al. Diagnostic value of tachypnoea in pneumonia defined radiologically. Arch Dis Child 2000;82:41-5.

27 Leng S, Tan RS, Chai KTC, et al. The electronic stethoscope. Biomed Eng Online 2015;14:66.

28 Pramono RXA, Bowyer S, Rodriguez-Villegas E. Automatic adventitious respiratory sound analysis: a systematic review. PLoS One 2017;12:e0177926.

29 Andrès E, Gass R, Charloux A, et al. Respiratory sound analysis in the era of evidence-based medicine and the world of medicine 2.0. J Med Life 2018;11:89.

30 Elhilali M, West JE. The stethoscope gets smart: engineers from Johns Hopkins are giving the humble stethoscope an Al upgrade. IEEE Spectr 2019;56:36-41.

31 West E, McLane I, McLane D. Introducing feelix, a digital stethoscope incorporating active noise control and automatic detection of lung sound abnormalities. J Acoust Soc Am 2019;145:1923-23.

32 Ramanathan A, Zhou L, Marzbanrad F. Digital stethoscopes in paediatric medicine. Acta Paediatrica 2018.

33 Kevat AC, Kalirajah A, Roseby R. Digital stethoscopes compared to standard auscultation for detecting abnormal paediatric breath sounds. Eur J Pediatr 2017;176:989-92.

34 Møller $\mathrm{H}$, Pedersen CS. Hearing at low and infrasonic frequencies. Noise Health 2004;6:37.
35 Thinklabs. Thinklabs one digital stethoscope. Centennial, CO, USA: Thinklabs, 2015. https://www.thinklabs.com/

36 Emmanouilidou D, McCollum ED, Park DE, et al. Adaptive noise suppression of pediatric lung auscultations with real applications to noisy clinical settings in developing countries. IEEE Trans Biomed Eng 2015;62:2279-88

37 McLane I, Emmanouilidou D, West JE, et al. Design and comparative performance of a robust lung auscultation system for noisy clinical settings. IEEE J Biomed Health Inform 2021;25:25832594.

38 IEEE. Validation of auscultation technologies using objective and clinical comparisons. 42nd Annual International Conference of the IEEE Engineering in Medicine \& Biology Society (EMBC), 2020.

39 Riaz BK, Ali L, Ahmad SA, et al. Community clinics in Bangladesh: a unique example of public-private partnership. Heliyon 2020;6:e03950.

40 McCollum ED, Park DE, Watson NL, et al. Listening panel agreement and characteristics of lung sounds digitally recorded from children aged 1-59 months enrolled in the pneumonia etiology research for child health (PERCH) case-control study. BMJ Open Respir Res 2017;4:e000193.

41 Flack VF, Afifi AA, Lachenbruch PA, et al. Sample size determinations for the two rater kappa statistic. Psychometrika 1988;53:321-5.

42 Ellington LE, Gilman RH, Tielsch JM, et al. Computerised lung sound analysis to improve the specificity of paediatric pneumonia diagnosis in resource-poor settings: protocol and methods for an observational study. BMJ Open 2012;2:e000506.

43 Scrafford C, Basnet S, Ansari I, et al. Evaluation of digital auscultation to diagnose pneumonia in children 2 to 35 months of age in a clinical setting in Kathmandu, Nepal: a prospective CaseControl study. J Pediatr Infect Dis 2016;11:028-36.

44 Allwell-Brown G, Hussain-Alkhateeb L, Sewe MO, et al. Determinants of trends in reported antibiotic use among sick children under five years of age across low-income and middle-income countries in 2005-17: a systematic analysis of user characteristics based on 132 national surveys from 73 countries. Int J Infect Dis 2021;108:473482.

45 Assembly UG. Political Declaration of the high-level meeting of the general assembly on antimicrobial resistance. New York, NY: United Nations, 2016.

46 Lynch T, Bialy L, Kellner JD, et al. A systematic review on the diagnosis of pediatric bacterial pneumonia: when gold is bronze. PLoS One 2010;5:e11989.

47 Hazir T, Nisar YB, Qazi SA, et al. Chest radiography in children aged 2-59 months diagnosed with non-severe pneumonia as defined by World Health organization: descriptive multicentre study in Pakistan. BMJ 2006;333:629.

48 McCollum ED, Park DE, Watson NL, et al. Digital auscultation in PERCH: associations with chest radiography and pneumonia mortality in children. Pediatr Pulmonol 2020;55:3197-208. 